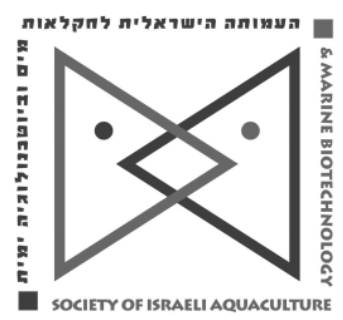

The IJA appears exclusively as a peer-reviewed on-line openaccess journal at http://www.siamb.org.il. To read papers free of charge, please register online at registration form. Sale of IJA papers is strictly forbidden.

\title{
Molecular Cloning, Characterization, and Expression Analysis of HSP60 in Mandarin Fish Siniperca chuatsi
}

\section{Pengfei Wang ${ }^{a b c}$, Peng $X u^{a c}$, Lei Zhou ${ }^{a c}$, Shuang Zeng ${ }^{a c}$, Guifeng $\mathbf{L i}^{\mathrm{ac} *}$}

a Key Laboratory for Aquatic Economic Animals, Institute of Aquatic Economic Animals, Guangdong Province, School of Life Sciences, Sun Yatsen University, Guangzhou, China, 510006

${ }^{b}$ Key Laboratory of South China Sea Fishery Resources Exploitation and Utilization, Ministry of Agriculture, and Laboratory of Fishery Ecology and Environment, Guangdong Province, The South China Sea Fisheries Research Institute, Chinese Academy of Fishery Sciences, Guangzhou, China, 510300

'South China Sea Bio-Resource Exploitation and Utilization Collaborative Innovation Center, Guangzhou, China 510006

Key words: Siniperca chuatsi; HSP60; heat shock; hypoxia; Aeromonas hydrophila

\begin{abstract}
HSP60 protein plays an important role in stress response, protein folding, and cell signaling. In this study, mitochondrial HSP6O from Siniperca chuatsi was identified, and its CDNA and gDNA structures, amino acid sequence features, and phylogenetic analysis, were described. Expression profiles during embryonic development, in different tissues and under stressful conditions were analyzed using RT-PCR. During embryogenesis, low levels of transcripts of ScHSP60 were detected during early developmental stages and were upregulated at blastopore closure stages to $1 \mathrm{dpf}$. ScHSP60 showed tissuespecific variation, highly expressed in ovaries under non-stressed conditions. Acute heat shock at $34^{\circ} \mathrm{C}$ resulted in strong upregulation of ScHSP6O in heart, liver, and head kidney in a time-dependent manner. However rapid and gradual elevated heat shock did not affect ScHSP60 expression when temperature reached $34^{\circ} \mathrm{C}$, although it was dramatically induced when temperature reached $38.8^{\circ} \mathrm{C}$. SCHSP6O was also markedly induced in the liver in a stage-dependent manner under hypoxia. Additionally, Aeromonas hydrophila infection augmented SCHSP6O in head kidney and spleen. Results showed that SCHSP6O expression is significantly modified under different environmental conditions including high temperatures, hypoxia, and bacterial infection. This study will further clarify the role of fish HSP6O in embryogenesis and under stressful conditions, and contribute to further investigation to understand stress tolerance and disease resistance of mandarin fish.
\end{abstract}

\footnotetext{
*Corresponding author: Guifeng Li Tel: +86 20 39332989; Fax: +862039332784

E-mail: liguif@mail.sysu.edu.cn
} 


\section{Introduction}

Heat shock proteins (HSPs) are highly conserved proteins that function as molecular chaperones to maintain cellular protein homeostasis under both normal physiological, and stress conditions, by facilitating folding of nascent polypeptides and degrading damaged proteins, assisting in intracellular trafficking, and modulating signal transduction as well as regulating immune responses (Roberts et al. 2010; Rupik et al. 2011). The HSP genes are generally divided into several major families: HSP100, HSP90, HSP70, HSP60, HSP40 and small HSPs based on molecular weight and sequence homology of proteins (Sarkar et al. 2011).

The $60 \mathrm{kDa}$ heat shock proteins (HSP60) are conserved in prokaryote and eukaryote cells and are required in the folding, translocation, and assembly of native proteins (Brocchieri and Karlin 2000). HSP60 is encoded by the chromosomal DNA and is typically active in the mitochondria; it has been found in organelles of endosymbiotic origin (Cheng et al., 1990). HSP60 cooperates with other chaperones such as HSP10 and HSP70, facilitates proper folding, assembly and transportation of protein complexes imported into mitochondria (Mayer 2010; Nakamura and Minegishi 2013). Apart from its classical chaperone function, mitochondrial HSP60 is also critically involved in the replication and transmission of mitochondrial DNA (Kaufman et al. 2003). HSP60 has been shown to be involved in stress response. The upregulation of HSP60 allows for maintenance of other cellular processes to protect cells from damage during stressful conditions (Vargas-Parada et al. 2001). Several studies have shown that HSP60 is necessary for cellular survival under toxic or stressful circumstances (Rossi et al. 2002). HSP60 also interacts with both the innate and adaptive immune system in mammals (Quintana and Cohen 2011).

To date, most studies of HSP60 have focused on mammals and typical model organisms, thus reports for teleost HSP60 is limited. HSP60 cDNA sequences from a few of fish species, including Ctenopharyngodon idellus (Xu et al. 2011), Tanichthys albonubes (Liu et al. 2011) and Epinephelus akaara (Qu et al. 2011), were cloned and similarity of amino acid structure to mammals suggested that fish HSP60 may function similarly to that in mammals. Mandarin fish, Siniperca chuatsi, an endemic freshwater fish species found in eastern Asian countries has been widely cultured in China. Various environmental stresses, including high temperature, hypoxia, and Aeromonas hydrophila infection, the causative agent of outbreaks of bacterial hemorrhagic septicemia disease, have frequently threatened the health of this fish, causing serious economic losses to the aquaculture industry (Lao et al. 2008; Chen et al. 2012). Understanding the characteristics of HSP60 expression under different conditions of stress will contribute to improving the stress tolerance and disease resistance of $S$. chuatsi as the response of the HSP60 gene to environmental stresses is totally unknown in S. chuatsi. In the present study we identified the HSP60 gene in S. chuatsi, and investigated its expression patterns during embryogenesis, in different tissues of juvenile fish, both under normal conditions and in response to heat shock, hypoxia, and bacterial infection.

\section{Materials and Methods}

Animals. Mandarin fish were obtained from BaiRong Aquatic breeding Co., Ltd (Guangdong, China). The fish (120 $\pm 20 \mathrm{~g}$, body weight, BW) were reared in a circulating water system containing a series of $2000 \mathrm{~L}$ tanks with water temperature of $25^{\circ} \mathrm{C}$ for more than two weeks in Sun Yat-Sen University, and fed fresh juvenile Cirrhinus molitorella at approximately $5 \%$ total biomass every day. Before tissue collection, fish were anesthetized with tricaine methanesulfonate (MS-222, XiangBo Biotec, China). Tissues were rapidly removed by team dissection, snap-frozen in liquid nitrogen, and stored at $-80^{\circ} \mathrm{C}$ until RNA extraction. All experiments were approved by the Animal Research and Ethics Committees of Sun Yat-Sen University and performed in accordance with the guidelines of the committee.

Embryo and tissue sampling. Embryos were obtained by artificial fertilization and hatching. Fertilized eggs were hatched in three vertical fish egg incubators (BaiRong Aquatic breeding Co., Ltd). Clean, oxygenated water (temperature $27^{\circ} \mathrm{C}$ ) flowed continuously into incubators. Pressure from below kept the eggs in a suspended state. 
Embryos at different stages including unfertilized eggs, fertilized eggs, 16-cell stage, morula, blastula, gastrula, closure of blastopore, appearance of myomere, tail-bud stage, muscle burl stage, blood circulating stage, crystal stage, pre-hatched larvae, 1-day posthatching (dph) and 7-dph larvae were collected and snap-frozen in liquid nitrogen. Tissues (brain, heart, gill, head kidney, liver, spleen, muscle, stomach, intestines, opisthonephros and ovary) from three female fish $(152-160 \mathrm{~g}$, BW) reared in nonstressed conditions were sampled and used for RT-PCR analysis of the tissue distribution of the two HSP90 isoforms.

Heat shock treatment. To investigate the expression patterns of ScHSP60 in response to thermal stress, the fish were subjected to three regimes of thermal stress: acute, rapidly elevated, and gradually elevated. For acute thermal stress, 20 fish (140 \pm $20 \mathrm{~g}, \mathrm{BW}$ ) maintained in water at $25^{\circ} \mathrm{C}$ were carefully and directly shifted to a tank of $2,000 \mathrm{~L}$ in the circulating water system at a constant temperature of $34^{\circ} \mathrm{C}$. Another 20 individuals were shifted to a tank of the same volume in the other circulating water system at $25^{\circ} \mathrm{C}$ and served as the control group. Six individuals from the treatment and control groups were randomly collected and sampled at 2, 6, and $12 \mathrm{~h}$, respectively. In the other two regimes of thermal stress, fish were transferred to the 2,000 L tank in the circulating water system (initial temperature: $25^{\circ} \mathrm{C}$ ); then the water temperature was elevated from 25 to $38.8^{\circ} \mathrm{C}$ at an average speed of $1.2^{\circ} \mathrm{C}$ increase per hour for rapid elevated thermal stress treatment, and $0.2^{\circ} \mathrm{C}$ increase per hour for gradual elevated thermal stress. Six fish were randomly sampled when the temperature reached $34^{\circ} \mathrm{C}$, six more at $38.8^{\circ} \mathrm{C}$, and another six individuals were maintained at $25^{\circ} \mathrm{C}$ (control group).

Hypoxia exposure. Forty-eight fish (132 $\pm 17 \mathrm{~g}$, BW) acclimated to the normal oxygen environment (dissolved oxygen, $\mathrm{DO}:>5.6 \mathrm{mg} / \mathrm{L}$, temperature: $25^{\circ} \mathrm{C}$ ) were randomly distributed into eight tanks of $400 \mathrm{~L}$ each $\left(A-D\right.$, and $\left.A^{\prime}-D^{\prime}\right)$ : A-C, exposed to a low oxygen level of $0.9 \pm 0.1 \mathrm{mg} / \mathrm{l}$ of $\mathrm{DO}$ for 2,6 and $12 \mathrm{~h}$, respectively; $D$, re-oxygenation under normal oxygen for $24 \mathrm{~h}$ after $6-\mathrm{h}$ hypoxia; $\mathrm{A}^{\prime}-\mathrm{D}^{\prime}$, exposed to normal oxygen, were referred to as control groups for A-D, respectively. Dissolved oxygen of $0.9 \pm 0.1 \mathrm{mg} / \mathrm{l}$ was maintained by controlling water and nitrogen inflows. Dissolved oxygen was monitored in real-time using a YSI Model 550A dissolved oxygen meter (Geo Scientific Ltd, USA).

Bacterial infection. Bacterial infection was conducted in a series of $400 \mathrm{~L}$ tanks with six individuals per tank. Sixty healthy fish (140 $\pm 15 \mathrm{~g}$, BW) were used for the challenge of virulent bacteria $A$. hydrophila, provided by MOE Key Laboratory of Aquatic Product Safety, Sun Yat-Sen University. Each fish was intraperitoneally injected with a total of $0.5 \mathrm{~mL}$ of $A$. hydrophila $\left(5 \times 10^{8} \mathrm{CFU} / \mathrm{mL}\right)$, which was diluted with sterile phosphate buffer solution (PBS, $\mathrm{pH} 7.4$ ). Another 30 fish injected with the same volume of aseptic PBS served as the control group. Six individuals from the treatment and control groups were randomly collected and tissues were sampled at $6,12,24,48$ and $72 \mathrm{~h}$ post injection, respectively.

Total RNA and genomic DNA isolation. Total RNA was extracted from tissues ground with liquid nitrogen using E.Z.N.A. total RNA kit II (Omega Bio-technology, USA) according to the manufacturer's instructions. Genomic DNA was isolated from the muscle using a TIANamp Genomic DNA Kit (Tiangen Biotec, China). Quality and quantity of RNA and DNA were assessed by the OD 260/OD280 measurement method and electrophoresis in $1 \%$ agarose gel.

Cloning of CDNAs and genomic DNAs. First-strand CDNA was synthesized from a pool of total RNA of liver and head kidney with the RT-PCR kit of first-strand CDNA synthesis (Invitrogen, Carlsbad, USA). Two pairs of degenerate primers, HSP60-F1/-R1 and HSP60F2/-R2 (Table 1), designed according to the highly conserved regions of HSP60 amino acid sequences from other fish species, were used to obtain the mandarin fish HSP60 cDNA fragments using nested PCR.

The technique of rapid amplification of CDNA ends (RACE) was used to obtain fulllength CDNA sequences. 3'-RACE PCR was performed with forward gene-specific primers for SCHSP6O (Table 1) and reverse primers anchor primer (AP) and abridged universal amplification primer (AUAP) in the 1 st and 2 nd round, respectively. The $5^{\prime}$-RACE PCR was 
performed with the template of purified first-strand CDNA which had a poly (C) end added to the $5^{\prime}$-terminal with a terminal deoxynucleotidyl transferase (TaKaRa, Japan), and the reverse gene-specific nested primers for HSP6O (Table 1) as well as the forward primers abridged anchor primer (AAP) and AUAP in the 1st and 2nd round, respectively.

Table 1. Primers and their applications in this study.

\begin{tabular}{llll}
\hline Primer name & Primer sequences $\left(5^{\prime}-\mathbf{3}^{\prime}\right)$ & Objective & \\
\hline HSP60-F1 & TGGCNGTCACNATGGGNCCRAA & 1st round intermediate & fragment \\
HSP60-R1 & CTGGCATNTCCTTCTCCTCCTTNG & amplification & \\
HSP60-F2 & GTNCAGGAYGTNGCCAACAACAC* & 2nd round intermediate & fragment \\
HSP60-R2 & GTCCTCACNACCTTNGTGGGRT* & amplification & \\
HSP60-3'F1 & CGGTTACATCTCCCCTTACTTCATC & 1st round 3'-RACE & \\
HSP60-3'F2 & AGAAGGACCGTGTGACAGATGCT & 2nd round 3'-RACE \\
HSP60-5'R1 & GAGCATCTGTCACACGGTCCTT & 1st round 5'-RACE \\
HSP60-5'R2 & GGTAGGCATCCTGGAACTCACACT & 2nd round 5'-RACE \\
g-HSP60-F & GTCGTTAGATCATTCCGTCTTGC & Genomic DNA cloning for HSP60 \\
g-HSP60-R & ACAGTGACACAATCCTGGCTAGG & \\
RT- HSP60-F & TGTCCCGTTCACCACGCTAT & Real-time quantitative \\
RT- HSP60-R & TTGATGGTTGATTGAACAGAGCC & PCR \\
18S-F & CTGAGAAACGGCTACCACATCC & Real-time quantitative & \\
18S-R & GCACCAGACTTGCCCTCCA & PCR \\
\hline
\end{tabular}

* $\mathrm{N}=\mathrm{G}+\mathrm{A}+\mathrm{C}+\mathrm{T} ; \mathrm{R}=\mathrm{G}+\mathrm{A} ; \mathrm{Y}=\mathrm{C}+\mathrm{T}$.

Amplifications for cloning the cDNAs were performed as follows: denaturation at $94^{\circ} \mathrm{C}$ for $3 \mathrm{~min}$, followed by $35-38 \mathrm{cycles}$ at $94^{\circ} \mathrm{C}$ for $30 \mathrm{~s}, 54-60^{\circ} \mathrm{C}$ for $30 \mathrm{~s}$ and $72^{\circ} \mathrm{C}$ for 1-2 min, and a final extension of $8 \mathrm{~min}$ at $72^{\circ} \mathrm{C}$. The amplified PCR products were purified with the TIANgel Midi Purification Kit (Tiangen Biotec, China) and cloned into a pEASY-T1 cloning vector using the TA cloning kit (TransGen Biotec). The positive recombinants were identified and sequenced.

Genomic DNA (gDNA) sequences coding SCHSP60 was amplified from total DNA by gene-specific primers gHSP60-F/-R, which were located at the terminus of $5^{\prime}$ - and $3^{\prime}-$ terminal untranslated regions (UTRs) of the CDNA sequences. PCR conditions for cloning of the genomic DNA were as follows: initial denaturation at $94^{\circ} \mathrm{C}$ for $3 \mathrm{~min}$, followed by 38 cycles of denaturation at $94^{\circ} \mathrm{C}$ for $30 \mathrm{~s}$, annealing at $58^{\circ} \mathrm{C}$ for $30 \mathrm{~s}$ and extension at $72^{\circ} \mathrm{C}$ for $8 \mathrm{~min}$, and a final extension of $10 \mathrm{~min}$ at $72^{\circ} \mathrm{C}$.

Sequences and phylogenic analysis. The search for nucleotide and amino acid sequence similarities was conducted with the BLAST programs at the National Center for Biotechnology Information. Multiple sequence alignments of amino acids were performed by ClustalX 2.1. Protein phylogenetic analysis was conducted with MEGA 6.0 using the neighbor-joining method with 1000 iterations. The molecular weight (Mw) and isoelectronic point (pI) of deduced amino acids were computed by $\mathrm{pI} / \mathrm{Mw}$ tool of ExPASy. The motifs of ScHSP60 were searched using the ScanProsite program, and signal sequences were searched using SignalP software.

Real-time quantitative PCR. Real-time quantitative PCR was used to quantify mRNA expression with a LightCycler $^{\circledR} 480$ II Real-Time PCR system (Roche) according to MIQE validation guidelines (Bustin et al., 2009). In the present study, 18S rRNA was selected as the reference gene for its stable expression in all situations. Specific primers (Table 1 ) were designed to amplify SCHSP60 and $18 S$ rRNA fragments. Total RNA was reversetranscribed using the PrimeScript RT reagent Kit with gDNA Eraser (TaKaRa, Japan), and cDNA equivalent to $10 \mathrm{ng}$ of total RNA was used for each reaction. Amplification was performed using SYBR ßPremix Ex Taq ${ }^{\mathrm{TM}}$ II (Tli RNaseHPlus) (TaKaRa, Japan) in 384-well plates. All reactions were carried out in three replicates, from which the mean threshold cycle $\left(\mathrm{C}_{\mathrm{T}}\right)$ values were calculated. Based on Tm values of the primer pairs, cycling conditions were designed as: initial denaturation step at $95^{\circ} \mathrm{C}$ for $30 \mathrm{~s}$, followed by 40 cycles of denaturation at $95^{\circ} \mathrm{C}$ for $5 \mathrm{~s}$, annealing at $58^{\circ} \mathrm{C}$ for $20 \mathrm{~s}$ and extension at $72^{\circ} \mathrm{C}$ for $30 \mathrm{~s}$, then a dissociation curve step $\left(95^{\circ} \mathrm{C}\right.$ for $1 \mathrm{~s}, 60^{\circ} \mathrm{C}$ for $20 \mathrm{~s}$, and $95^{\circ} \mathrm{C}$ for $1 \mathrm{~s}$ ) was conducted to evaluate the specificity.

Ten fold serial dilutions of recombinant plasmid pEASY-T1 containing ScHSP6O and $18 S$ rRNA genes were used to construct standard curves. Amplification efficiencies (E) were determined by the equation $\mathrm{E}=10\left({ }^{-1 / \mathrm{Slope}}\right)-1$. Here, amplification efficiencies of SCHSP6O and $18 S$ rRNA were 98.8 and $104.6 \%$, respectively. 
Statistical analysis. The relative transcript levels of ScHSP60 were normalized to the level of $18 S$ rRNA in the same sample by the $2^{-\Delta \mathrm{Ct}}$ method and the relative expression levels to the control were determined by the $2^{-\Delta \Delta \mathrm{ct}}$ method. Statistical analyses were performed using IBM SPSS software version 22. Data presented in this study were analyzed using one-way ANOVA followed by Tukey's test and expressed as mean \pm SD.

\section{Results}

Cloning and sequence analysis of ScHSP60 CDNA. The full length of ScHSP60 cDNA sequence was $2505 \mathrm{bp}$, containing an open reading frame (ORF) of $1731 \mathrm{bp}$, a 5'-UTR of $85 \mathrm{bp}$ and a 3'-UTR of $689 \mathrm{bp}$ with a polyadenylation signal (AATTAAA) located 13 nucleotides upstream from the poly $(A)$ tail. The deduced amino acid sequence encoded a protein of 576 amino acids with calculated molecular weight of $61.11 \mathrm{kDa}$ and theoretical isoelectric point of 5.56. The cDNA sequence of ScHSP6O was deposited in the GenBank database with accession number KU997668.

As shown in Fig. 1, ScHSP60 sequence contains the classical mitochondrial HSP60 signature motif AAVEEGIVPGGG (amino acid, aa, 430-441) and a typical GGM repeat site at the $C$ terminus. Using SignalP 4.1 Server, a presequence of 28 amino acids at the $N$ terminus was predicted that is required for import into the mitochondria. A conserved domain search on the NCBI database identified a conserved ATP-binding/Mg2 ${ }^{+}$-binding site (aa, 195-213).



CTTCACCAAACTCTGCCCACACCGACGGACTGACTGGCCATGAGTATGTTGGAGCTGCAAATGGTTGATGACCCCCTCCCCTCCCCACTA ACTTCACACGATTCTGCCTCCTTCCTCTTTGTCTGGGTTTCTGGAGGAGCCTGACTCCGTGCCCCTCTGTCCCGTTCACCACGCTATGGG AGCCCATCAAGCCCAATTACACCACAGTCCCCTTCACACCTCTAATGGCTCTGTTCAATCAACCATCAACATCTGCACAAAACTCATGTG AAACACTCAACAGAAAGAAGAATGCTTATTCAGATTTACGTACAGATTTGATCTCTGTAAGAAGTAGTAAAGTGGTCTAAGAAGAAACTA ACATAGTACACATAATGATGACGCTCTATTACTGCAAAGAGAGCAGGGCTTGAACGAGAAAAGATGCCTAGCCAGGATTGTGTCACTGTC CTITATTTTIGTCATAGTATTTTGTTAATTGTTTTGTTTTITTATATATATATATACATCTGTCATGTTTTGTTTCCCCCAGCTAATATA TTATGTGGGTTAAAATCTGTCATTTGATTT AATTAAACCGATTTTTTCTTAAAAAAAAAAAAAAAAAAAAAAAAAA

Fig.1 Nucleotide sequence and deduced amino acid sequences of S.chuatsi HSP60. The polyadenilation signal at $\mathrm{N}$ terminus is shaded and the GGM repeat site at $\mathrm{C}$ terminus is boxed. The typical mitochondrial HSP60 signature motif is boxed and shaded. The ATPbinding $/ \mathrm{Mg}^{+}$ binding site is underlined, and the polyadenylation signal is double lined. 
Sequence identities and phylogenetic analysis of ScHSP60. The homology analysis based on the amino acid sequences revealed that ScHSP60 displayed highly identity (> $90 \%$ ) with its counterparts in other fish species, especially Epinephelus coioides, up to $95.5 \%$. The ScHSP60 also shared more than $86 \%$ identities with its orthologs in birds and mammals, and $74.9 \%$ identities with Bactrocera dorsalis (Table 2).

Table 2. Identity analysis of HSP60 amino acid sequences from vertebrates. Data are expressed as percentage of amino acid sequence identity.

\begin{tabular}{|c|c|c|c|c|c|c|c|c|c|c|c|c|c|}
\hline 1 & 2 & 3 & 4 & 5 & 6 & 7 & 8 & 9 & 10 & 11 & 12 & & \\
\hline & 95.5 & 94.8 & 94.4 & 94.4 & 93.6 & 90.9 & 91.5 & 84.5 & 87.9 & 86.0 & 74.9 & 1 & S. chuatsi \\
\hline & & 96.5 & 95.5 & 96.5 & 95.3 & 91.0 & 92.3 & 84.9 & 88.4 & 86.8 & 74.4 & 2 & E. coioides \\
\hline & & & 96.5 & 95.8 & 96.0 & 90.6 & 91.8 & 85.2 & 88.8 & 86.7 & 74.6 & 3 & C. variegatus \\
\hline & & & & 96.0 & 94.6 & 90.8 & 91.6 & 85.6 & 90.0 & 87.4 & 74.6 & 4 & O. niloticus \\
\hline & & & & & 95.1 & 90.4 & 91.6 & 85.7 & 88.8 & 87.0 & 74.7 & 5 & C. striata \\
\hline & & & & & & 90.8 & 91.8 & 85.7 & 88.2 & 87.4 & 74.4 & 6 & K. marmoratus \\
\hline & & & & & & & 96.2 & 84.3 & 87.7 & 85.4 & 73.4 & 7 & T. albonubes \\
\hline & & & & & & & & 86.1 & 88.9 & 87.7 & 74.1 & 8 & D. rerio \\
\hline & & & & & & & & & 90.4 & 90.2 & 75.7 & 9 & X. laevis \\
\hline & & & & & & & & & & 93.9 & 75.7 & 1 & G. gallus \\
\hline & & & & & & & & & & & & $\mathbf{0}$ & \\
\hline & & & & & & & & & & & 75.3 & 1 & H.sapiens \\
\hline & & & & & & & & & & & & 1 & \\
\hline & & & & & & & & & & & & 1 & dorsalis \\
\hline
\end{tabular}

To evaluate the evolutionary relationship of HSP60s of mandarin fish and other species, we constructed a phylogenetic tree using software MEGA 6.0 by the neighborjoining method (Fig. 2). According to the phylogenetic tree, vertebrates were divided into two groups based on HSP60s. The mammals, birds and amphibians were clustered into one group, the teleosts were separated and clustered into another group. In addition, the teleosts were further divided into two subgroups supported statistically (Bootstrap value $=100 \%$ ). Species from Perciformes and Cyprinodontiformes were clustered with a subgroup containing the ScHSP60 identified in this study, and species from Cypriniformes were clustered with the other subgroup. 




Fig. 2 Phylogenetic tree of the vertebrate HSP60 proteins constructed with MEGA 6.06 software using neighbor-joining method. Bootstrap support values $(1,000$ replicates) of greater than $50 \%$ are indicated at the nodes. Scale bar indicates an evolutionary distance of 0.02 amino acid substitution per position in the sequence. Sequence database accession numbers in GenBank are indicated in parentheses.

Cloning and sequence analysis of ScHSP60 gDNA. Using specific primers close to the terminus of full-length CDNA, SCHSP60 genomic sequence was obtained and deposited in GenBank under the accession number KU997669. The coding region of ScHSP6O contains 10 exons $(174,336,96,94,169,100,246,175,179$ and 162 bp) interrupted with 9 introns (155, 103, 108, 152, 159, 130, 95, 1271 and 1462 bp). ScHSP60 gene contains an intron (length $1538 \mathrm{bp}$ ) within its $5^{\prime}$-UTR. All the introns obey the GT-AG rule. Alignment of genomic sequences of HSP60s in $S$. chuatsi and other vertebrates reported in Ensemble database showed that the genomic structure of HSP60 is highly conservative (Fig. 3). The genomic sequences of coding regions of fish HSP60s all consist of 10 exons interrupted with 9 introns. In comparison with fish species, an extra intron was insert in the second exon in Amphilia (Xenopus laevis), birds (Gallus gallus) and mammals (Homo sapiens). 


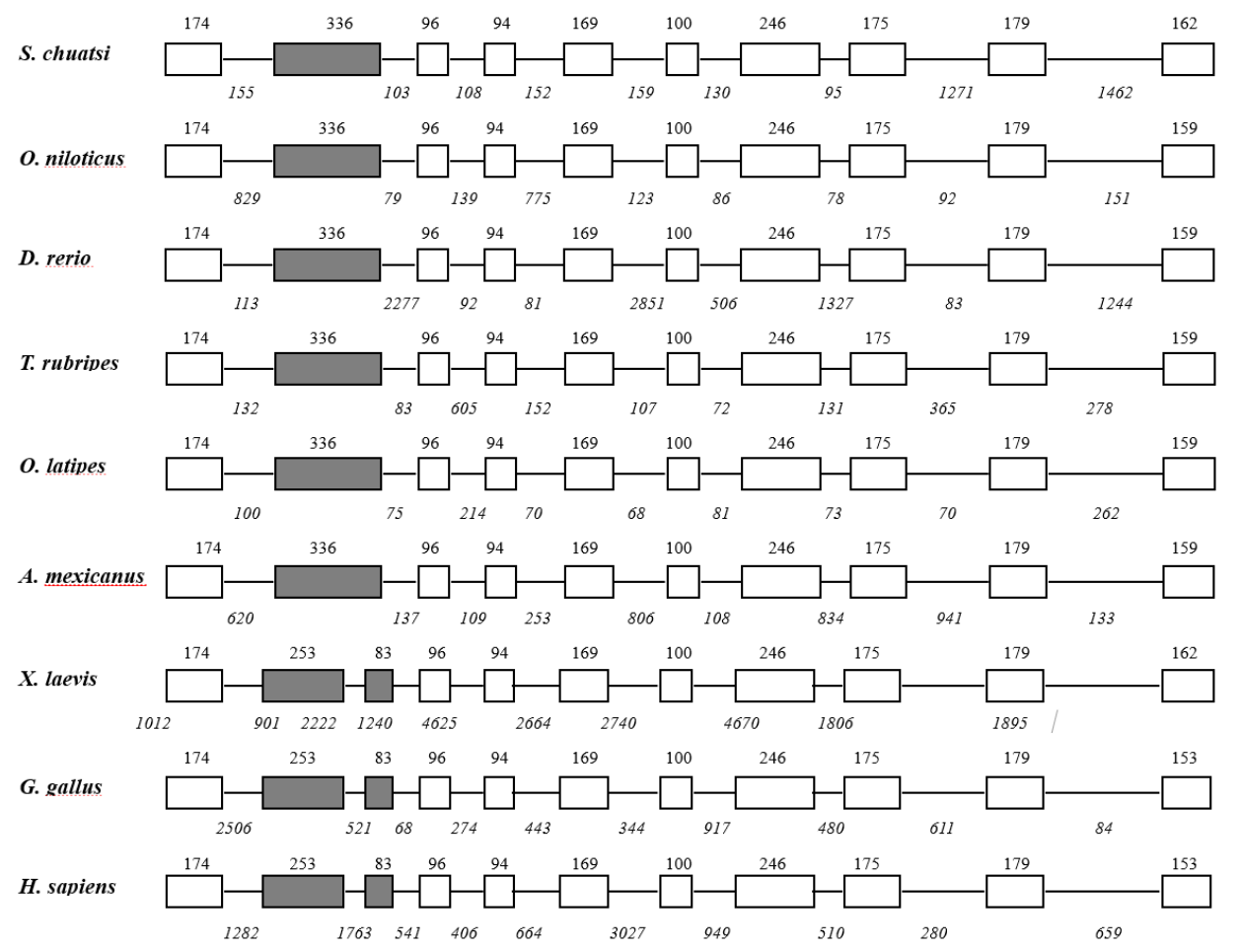

Fig. 3 Comparison of genomic structures of HSP60 in S. chuatsi and other vertebrates, including Oreochromis niloticus, Danio rerio, Takifugu rubripes, Xiphophorus maculatus, Oryzias latipes, Astyanax mexicanus, Xenopus laevis, Gallus gallus and Homo sapiens. The rectangles represent exons, and the lines indicate introns. Figures above the rectangles and below the lines represent length (bp) of exons and introns, respectively.

\section{Expression patterns of ScHSP60 during embryonic development}

SCHSP60 transcripts could be detected throughout the embryonic developmental stages (Fig. 4). The transcript levels of SCHSP6O had no significant changes from fertilized eggs to gastrula, but were significantly increased from the stage of closure of blastopore to 1 $\mathrm{dph}$. The highest level appeared at metameres appearance stage and muscle burl stage, approximately 4 times $(P<0.05)$ of that in the fertilized eggs.

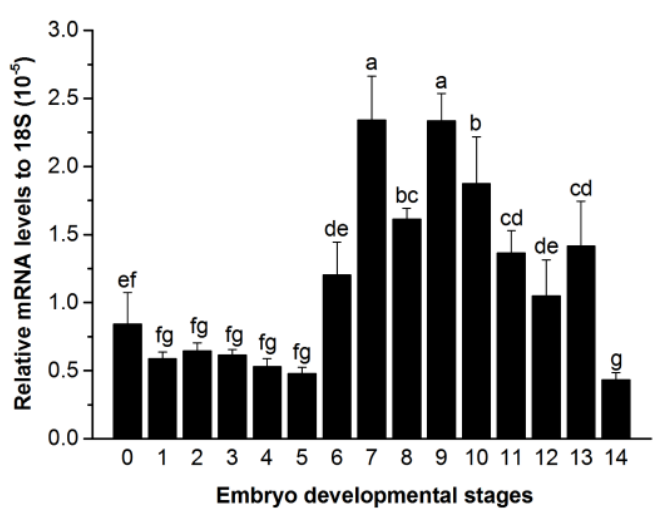

Fig. 4 Expression patterns of HSP60 during embryonic developmental stages of S.chuatsi. Each bar represents the mean \pm SD of three samples. Different letters above the bars indicate significant difference $(P<0.05) .0$ : Unfertilized eggs; 1, Fertilized eggs; 2, 16-cells stage; 3, Morula; 4, Blastula; 5, Gastrula; 6, Closure of blastopore; 7, Metameres appearance; 8, Tail-bud stage; 9, Muscle burl stage; 10, Crystal stage; 11 , Blood circulating stage; 12, pre-hatched larvae; 13, 1 dph larvae; 14, 7 dph larvae.

Tissue expression of ScHSP60. Under normal physiological conditions, the highest level of ScHSP60 was found in the tissue-specific expression profile of the ovaries. In nongonadal tissues, ScHSP60 was highest in opisthonephros, followed by brain, head kidney, and spleen; lowest level was found in muscle, only $1.7 \%$ of that in opisthonephros (Fig.5). 


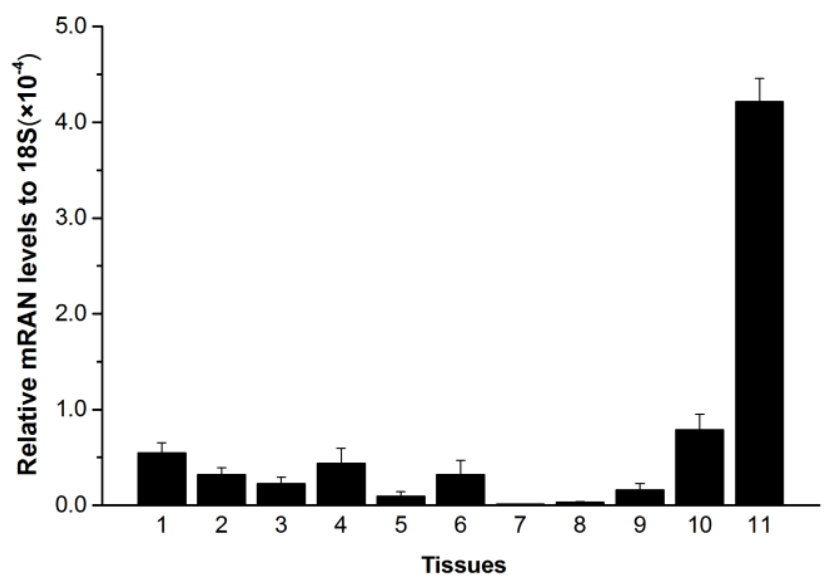

Fig.5 The mRNA expression of HSP70 family members in various tissues of Siniperca chuatsi. The mRNA expression was quantified by real-time RT-PCR and normalized with $18 S$ rRNA. Data presented are expressed as mean $\pm S D, \mathrm{n}=3$ fish. 1 : Brain; 2: Heart; 3: Gill; 4: Head kidney; 5: Liver; 6: Spleen; 7: Muscle; 8: Stomach; 9: Intestines; 10: Opisthonephros; 11: Ovary.

Expression patterns of ScHSP60 after thermal stress exposure. To investigate the expression patterns of ScHSP60 after heat shock exposure, the fish were exposed to three regimes of heat shock: acute, rapid elevated and gradual elevated heat shock. The experimental temperatures in the three regimes were based on the fact that $S$. chuatsi has a natural temperature range of between 15 and $32{ }^{\circ} \mathrm{C}$ with an upper lethal limit of $34^{\circ} \mathrm{C}$ under acute heat stress, and approximately $39^{\circ} \mathrm{C}$ under rapid and gradual heat shocks measured in the present study. The expression levels of ScHSP6O in heart, liver and head kidney under acute heat shock exposure are shown in Fig. 6a. The results showed that acute heat shock at $34^{\circ} \mathrm{C}$ significantly induced the expression of ScHSP6O in these tissues. In heart, heat shock for $6 \mathrm{~h}$ resulted in the highest up-regulation of SCHSP60, 11.7-times that of the control. In liver and head kidney, heat shock for $2 \mathrm{~h}$ resulted in the highest up-regulation by averages of 2.8- and 2.6-times that of in controls. And when heat shock for $12 \mathrm{~h}$, the ScHSP60 levels began to recover towards its baseline in heart and head kidney, and recovered and even below the baseline in liver.

The expression levels of SCHSP6O in heart under fast elevated heat shock (elevating temperature rate: $\sim 1.2^{\circ} \mathrm{C} / \mathrm{h}$ ) and gradual elevated heat shock (elevating temperature rate: $\sim 0.2{ }^{\circ} \mathrm{C} / \mathrm{h}$ ) were also investigated (Fig. $6 \mathrm{~b}$ ). Under fast elevated heat shock from 25 to $34^{\circ} \mathrm{C}$, the mRNA level of ScHSP60 was increased by averages of 3.1 times, which continued to be increased with the temperature elevating to $38.8^{\circ} \mathrm{C}$, by averages of $18.6{ }^{\circ} \mathrm{C}$ 。 Likewise, gradual elevated heat shock also resulted in elevation of SCHSP60, but was lower than those under rapid elevated heat shock, by averages of 2.4 times at $34{ }^{\circ} \mathrm{C}$ and 16.7 - times at $38.8^{\circ} \mathrm{C}$.

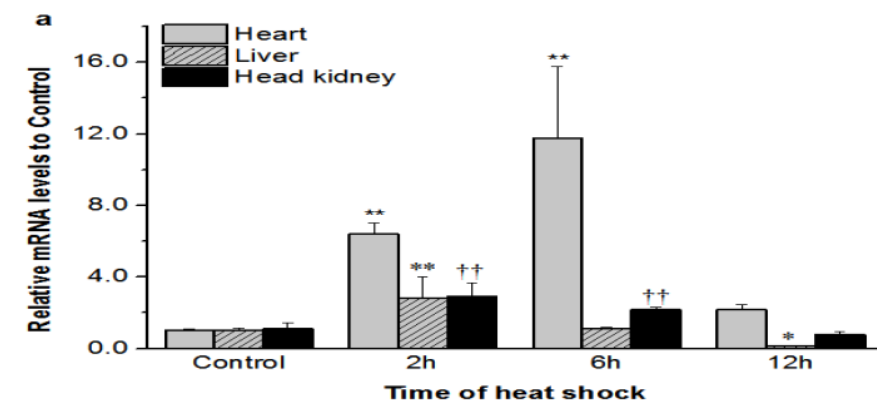

Fig. 6 Expression of S. chuatsi HSP6O during three regimes of heat shock exposure. a, acute heat shock: fish maintained at $25^{\circ} \mathrm{C}$ were carefully and directly shifted to $34^{\circ} \mathrm{C}$. b, rapid elevated and gradual elevated heat shock: The elevating temperature rate in the rapid elevated and gradual elevated heat shock regimes were $1.2^{\circ} \mathrm{C} / \mathrm{h}$ and $0.2^{\circ} \mathrm{C} / \mathrm{h}$ from $25^{\circ} \mathrm{C}$, respectively. The mRNA levels were measured by real-time RT-PCR and normalized by $18 S$ rRNA. The fish that maintained at $25^{\circ} \mathrm{C}$ over the entire experimental period were taken as the control group. Data presented are expressed as mean \pm SD $(n=6)$. Symbols of "*"/"*" / "†" $(P<0.05)$ and "**" / "**"/"十+" $(P<0.01)$ above the bars represent significant difference. 
Expression of ScHSP60 after hypoxia exposure. In order to determine whether hypoxia influences the expression of SCHSP60, its mRNA levels in response to hypoxia in heart (data not shown) and liver (Fig. 7) were detected. Since there were no significant changes in the mRNA levels of ScHSP60 throughout the experiment, the average expression level was used as a control. Although SCHSP6O was not significantly changed in the heart, the mRNA level was markedly increased in the liver by hypoxia in a timedependent manner, increasing 1.9 and 4.6 times compared with the control group at 6 and $12 \mathrm{~h}$, respectively. In addition, the induced ScHSP60 mRNA level after 6h-hypoxia exposure returned to its baseline during re-oxygenation over $24 \mathrm{~h}$.



Fig.7 Expression of HSP60 in liver of S. chuatsi in response to hypoxia and re-oxygenation. The mRNA levels in the time-course were qualified by RT-PCR and normalized by $18 \mathrm{~S} R N A$. After $6 \mathrm{~h}$ of hypoxia the DO levels were adjusted back to normoxic levels within 20 min and fish were sampled after $24 \mathrm{~h}$ of re-oxygenation $(\mathrm{Re})$. Data presented are expressed as mean \pm SD $(n=6)$. Asterisk $(\mathrm{P}<0.05)$ and double asterisks $(P<0.01)$ above the bars indicate significant difference compared with the control


group.

Expression of SCHSP60 after A. hydrophila infection. The expression patterns of ScHSP60 in head kidney and spleen from 6 to $72 \mathrm{~h}$ post-infection with $A$. hydrophila were examined by real-time quantitative PCR (Fig. 8). Results showed that infection with $A$. hydrophila significantly $(P<0.01)$ increased the SCHSP6O expression in the head kidney and spleen. During the time-course experiment, ScHSP60 mRNA level initially increased significantly and reached the highest level of 3.2 times in the head kidney at $12 \mathrm{~h}$ and gradually decreased to baseline thereafter. Similarly, its level in the spleen was markedly increased at $6 \mathrm{~h}$, up to 7.7 times of that in the control group, and then decreased gradually, returning to the baseline at $48 \mathrm{~h}$.

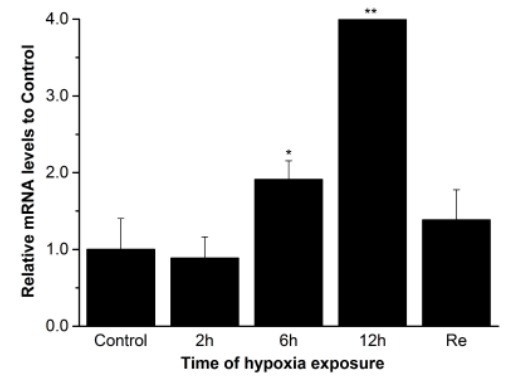

Fig. 8 Expression of SCHSP6O in the head kidney and spleen after A.hydrophila infection. The mRNA levels in the time-course study were qualified by RT-PCR and normalized by $18 S$ rRNA. Data presented are expressed as mean \pm SD $(n=6)$. Single asterisk $(P<0.05)$ and double asterisks $(P<0.01)$ above the bars indicate significant difference compared with the control group.

\section{Discussion}

HSP60 is a molecular chaperone located mainly in the mitochondria. In the present study, the HSP60 cDNA and gDNA from mandarin fish was identified and characterized. The full length of ScHSP6O cDNA is 2505 bp with an ORF of 1731 bp encoding 576 amino acids. ScHSP60 sequences have three major functional domains: the apical domain, the equatorial domain, and the intermediate domain (Brocchieri and Karlin 2000). ScHSP60 has the classical mitochondrial HSP60 signature motif and a mildly hydrophobic conserved GGM repeat at C-terminal that are required for the rapid folding of some proteins (Tang et al. 2006). Sequence alignment shows ScHSP60 is highly homologous 
with HSP60 genes from other vertebrates. The phylogenetic analysis revealed that ScHSP60 was clustered with its counterparts in other fish species. Alignment of gDNA sequences of HSP60s from S. chuatsi and other vertebrates reported in Ensemble database revealed that numbers of nucleotide bases of all corresponding exons of these fish species are identical except for one; S. chuatsi has 3 additional nucleotide bases, more than other fish, encoding one more Gly in the Gly-Gly-Met belonging to the GGM repeat, probably derived from slipped-strand mispairing during gene duplication. On the basis of CDNA and gDNA characterization, multiple alignment, and phylogenetic analysis, we were able to confirm that the newly identified ScHSP60 is a member of the mitochondrial HSP60 family.

To date there has been limited information regarding HSP60 in embryonic development of teleosts. In the present study, we had ScHSP6O transcripts in unfertilized eggs and embryos before the midblastula transition (MBT) period, until activation of zygotic transcription (Kane and Kimmel 1993), suggesting maternal heredity. ScHSP6O expression significantly increased from the stage of blastopore closure to $1 \mathrm{dph}$, at high levels at metameres appearance stage, and muscle burl stage, indicating that mitochondrial HSP60 plays an important part in embryonic development of mandarin fish. This was consistent with the brief summary of HSP60 at significantly higher levels at early stages of oocyte differentiation in teleosts (Lubzens et al. 2010). However, in a study of HSP60 of C. idella, maternal original CiHSP60 transcripts were reduced markedly at the gastrula stage and kept at low levels of less than 1/10 of the fertilized eggs (Xu et al. 2011). Almost the opposite expression patterns of HSP60 between C. idella and $S$. chuatsi may be due to species variance, and more studies on HSP60 expression during embryonic development are needed on other fish species.

SCHSP60 showed a tissue-specific variation pattern in different tissues under normal conditions. The much higher expression level in the ovaries (phase III during ovarian development) indicated that ScHSP6O may play a crucial physiological role in ovary development. In addition, relatively high levels of SCHSP6O were found in head kidney and spleen, two important immune organs for mandarin fish. Similar results were also found in C. idella HSP60 (Xu et al. 2011), suggesting that HSP60 may be related to immune function.

Temperature is a major factor for survival of animals. One major type of cellular damage resulting from heat stress is denaturation of proteins, and adaptation to this stress leads to upregulation of heat shock proteins, which interact with stress-denatured proteins to maintain or restore their native structure and prevent aggregation and degradation (Purohit et al. 2014). In our previous study, we reported elevated mandarin fish HSP70 when exposed to heat shock (Wang et al. 2014). SCHSP60 was also upregulated during three regimes of thermal stress: acute, fast, and gradual heat shock. It has been demonstrated that heat stress induces apoptosis through activation of reactive oxygen free radicals (ROS) (Pallepati and Averill-Bates 2011), and HSP60 can inhibit the generation of ROS (Magnoni et al. 2014). HSP60 may inhibit cell apoptosis by suppressing ROS generation caused by heat stress. In addition, compared with elevation under acute shock at $34^{\circ} \mathrm{C}, \mathrm{ScHSP} 60$ expression strongly upregulated at $38.8^{\circ} \mathrm{C}$ and did not significantly change at $34^{\circ} \mathrm{C}$ when exposed to rapid or gradual heat shock, suggesting that induction of $H S P 60$ by heat shock requires drastic changes of temperature or large temperature differences.

There is sparse information of HSP60 expression under hypoxia in fish. In mammals, mice HSP6O was up-regulated in hypoxic preconditioning and ischemia, and involved in the development of hypoxic preconditioning (Jiang et al. 2011). In this study, the expression of ScHSP60 in liver significantly increased with 6-12 h of hypoxia, suggesting it is also involved in the cellular process of hypoxic response.

HSP60 is a node in intracellular molecular networks, and is also a linking molecule in intercellular immune networks (Quintana and Cohen 2011). Inside both prokaryotic and eukaryotic cells, HSP60 functions as a molecular chaperone coming into contact with most intracellular proteins (Borges and Ramos 2005). In the extracellular environment, HSP60 alone or combined with microbial proteins acts as a connecting link between 
immune cells to coordinate activity of the immune system (Quintana and Cohen 2011). Most studies on immune functions of HSP60 have focused on mammals. There are few reports regarding HSP60 in fish immune response. The expression of HSP60 in the liver, gills and spleen of E. akaara, was upregulated when infected with Vibrio (Qu et al. 2011). HSP60 expression in multiple tissues including liver, heart, intestine, head kidney, spleen, and gills of grass carp was significantly increased after 3 days of infection by $A$. hydrophila (Xu et al. 2011). Our study showed that the different stages of development affected upregulation of SCHSP6O in the head kidney and spleen. In the head kidney, HSP60 reaction manifested primarily in the early stage of infection, while in the spleen, HSP60 mRNA was higher during long-term infection. These results suggest that ScHSP60 stimulated immune response to protect fish from damage caused by $A$. hydrophila.

In summary, HSP60 cDNA and gDNA were identified and characterized in mandarin fish S. chuatsi. Characteristic amino acid sequence features, DNA structure, and phylogenetic analysis showed that SCHSP60 belongs to the mitochondrial HSP60 family. Furthermore, mRNA expression profiles of ScHSP60 during embryonic development, in different tissues and after exposure to different environmental stresses were analyzed using real-time RT-PCR. Expression profiles found in this study suggest that ScHSP60 is involved in embryogenesis and the stress responses of high temperature, hypoxia, and bacterial infection. The identification and expression analysis of ScHSP60 will benefit to further clarify the important roles of HSP60 in embryogenesis, complex environmental, physiological, and stressful conditions in aquatic vertebrates, and contribute to further studies to enhance stress tolerance and disease resistance of mandarin fish.

\section{Acknowledgements}

This research was funded by Foshan Innovative and Entepreneurial Research Team Program (No.2014IT100122), The Science and Technology Projects of Guangdong Oceanic and Fisheries (No.A201401A02), The Science and Technology Planning Projects of Guangdong Province (No.2014A020208022, No. 2012A020800001, No.2015A030310253 and No. 2016A030303029), Special Fund for Agro-scientific Research in the Public Interest, China (No.201303048), and Modern Agriculture Talents Support Program(2016-2020), Chinia.

\section{References}

Borges J. C., and C. H. Ramos, 2005. Protein folding assisted by chaperones. Protein Pept Lett, 12 (3):257-61.

Brocchieri L., and S. Karlin, 2000. Conservation among HSP60 sequences in relation to structure, function, and evolution. Protein Sci, 9 (3):476-86.

Chen W.X., Liang X.F., Fu Y, and W. Ye, 2012. The Plankton Composition in Mandarinfish Siniperca chuatsi Ponds and Bait Fish Ponds in Hot Season. Chinese Journal of Fisheries, 25 (1):25-29.

Cheng M.Y., HartI F.U., and A.L. Horwich, 1990. The mitochondrial chaperonin hsp60 is required for its own assembly. Nature, 348:455-8.

Jiang S.J., Zhang N., and J.F. Li, 2011. CPKCY-HSP60 signal pathway participates in hypoxic preconditioning in mice. Chin Pathophysio, 6:1066-70.

Kane D. A., and C. B. Kimmel, 1993. The zebrafish midblastula transition. Development, 119 (2):447-56.

Kaufman B. A., Kolesar J. E., Perlman P. S., and R. A. Butow, 2003. A function for the mitochondrial chaperonin $\mathrm{Hsp60}$ in the structure and transmission of mitochondrial DNA nucleoids in Saccharomyces cerevisiae. J Cell Biol, 163 (3):457-61.

Lao H. H., Sun Y. N., Yin Z. X., Wang J., Chen C., Weng S. P., He W., Guo C. J., Huang X. D., Yu, X. Q., and J. G. He, 2008. Molecular cloning of two C1q-like cDNAs in mandarin fish Siniperca chuatsi. Vet Immunol Immunopathol, 125 (1-2):37-46.

Liu H.C, Chen H.H., Qin J.H., and X.F. Ma., 2011. Cloning and expression of heat shock protein 60 cDNA of Tanichtys albonubes. J Huazhong Agric Univ 30:635-9.

Lubzens E., Young G., Bobe J., and J. Cerda, 2010. Oogenesis in teleosts: how eggs are formed. Gen Comp Endocrinol, 165 (3):367-89.

Magnoni R., Palmfeldt J., Hansen J., Christensen J. H., Corydon T. J., and P. 
Bross, 2014. The Hsp60 folding machinery is crucial for manganese superoxide dismutase folding and function. Free Radic Res, 48 (2):168-79.

Mayer M. P, 2010. Gymnastics of molecular chaperones. Mol Cell, 39 (3):321-31.

Nakamura H., and H. Minegishi, 2013. HSP60 as a drug target. Curr Pharm Des, 19 (3):441-51.

Pallepati P., and D. A. Averill-Bates, 2011. Mild thermotolerance induced at 40 degrees $C$ protects HeLa cells against activation of death receptor-mediated apoptosis by hydrogen peroxide. Free Radic Biol Med, 50 (6):667-79.

Purohit G. K., Mahanty A., Suar M., Sharma A. P., Mohanty B. P., and S. Mohanty, 2014. Investigating hsp gene expression in liver of Channa striatus under heat stress for understanding the upper thermal acclimation. Biomed Res Int, 2014.DOI 10.1007/s10695-016-0202-x.

Qu M, Shi X.F, Zhang Z.W., and S.X., Ding, 2011. Cloning of HSP60 gene from Epinephelus akaara and its express characterization before and after vibrionic stressed. Acta Oceanol Sin, 33:111-20.

Quintana F. J., and I. R. Cohen, 2011. The HSP60 immune system network. Trends Immunol, 32 (2):89-95.

Roberts R. J., Agius C., Saliba C., Bossier P., and Y. Y. Sung, 2010. Heat shock proteins (chaperones) in fish and shellfish and their potential role in relation to fish health: a review. J Fish Dis, 33 (10):789-801.

Rossi M. R., Somji S., Garrett S. H., Sens M. A., Nath J., and D. A. Sens, 2002. Expression of hsp 27, hsp 60, hsc 70, and hsp 70 stress response genes in cultured human urothelial cells (UROtsa) exposed to lethal and sublethal concentrations of sodium arsenite. Environ Health Perspect, 110 (12):1225-32.

Rupik W, Jasik K, Bembenek J, Widlak W, 2011. The expression patterns of heat shock genes and proteins and their role during vertebrate's development. Comp Biochem Physiol A Mol Integr Physiol, 159:349-66.

Sarkar S, Singh MD, Yadav R, Arunkumar KP, Pittman GW, 2011. Heat shock proteins: Molecules with assorted functions. Frontiers in Biology, 6:312-27.

Tang Y. C., Chang H. C., Roeben A. , Wischnewski D., Wischnewski N., Kerner M. J., Hartl F. U., and M. Hayer-Hartl, 2006. Structural features of the GroEL-GroES nano-cage required for rapid folding of encapsulated protein. Cell, 125 (5):903-14.

Vargas-Parada, L., Solis C. F., and J. P. Laclette, 2001. Heat shock and stress response of Taenia solium and T. crassiceps (Cestoda). Parasitology, 122 (Pt 5):583-8.

Wang P.F, Zeng S, Xu P, Zhou L, Zeng L, Lu X, Wang H.F, and G. F. Li, 2014. Identification and expression analysis of two HSP70 isoforms in mandarin fish Siniperca chuatsi. Fisheries Sci, 80:803-17.

Xu, X. Y., Shen Y. B., Fu J. J., Liu F., Guo S. Z., Yang X. M., and J. L. Li. 2011. Molecular cloning, characterization and expression patterns of HSP60 in the grass carp (Ctenopharyngodon idella). Fish Shellfish Immunol, 31 (6):864-70. 